\title{
Artistes visuelles et sorcellerie : de la magie comme instrument créatif de lutte politique Ophélie Naessens ${ }^{1}$
}

\section{Des artistes et des sorcières : réappropriation(s) d'une figure d'émancipation}

Nombreuses sont les artistes contemporaines qui convoquent l'iconographie de la sorcellerie. Certaines s'emploient à réécrire ses histoires passées, tandis que d'autres explorent ses histoires actuelles. C'est le cas par exemple de Camille Ducellier dans son film Sorcières, mes sœurs (2010), dont le synopsis précise : "Sorcières, hameçons du diable, tisons d'enfer... Quelles sont ces femmes qui incarnent le danger des époques ? Féministes pour sûr, souterraines parfois et singulières dans leur démarche, voilà quelques sorcières d'aujourd'hui, qui ont croisé mon regard ${ }^{2} . » D^{\prime}$ 'autres réactivent la figure de la sorcière, telle Tatiana Karl Pez en maîtresse de cérémonie officiant au clair de Lune dans la performance Circle Danse La Luna $^{3}$ (2018). D'autres encore actualisent des pratiques et des gestes, présentant des rituels ancestraux ou inédits, telle la plasticienne franco-gabonaise Myriam Mihindou qui réalise des performances pensées comme des rituels à l'ambition curative, des transes reliant l'être au monde qui l'entoure, dans une tentative de réparation et de transcendance de la blessure (No Sensibility, 2013). Ces artistes puisent ainsi dans les imaginaires des mondes invisibles, des métamorphoses, du mystère, de la mystique et de la magie.

Au-delà du folklore et de l'ésotérisme, la sorcière est pour ces plasticiennes l'incarnation d'une émancipation féminine au potentiel subversif; le symbole d'une femme forte et puissante. La sorcière personnifie la révolte, s'élevant contre les accusations d'irrationalité, les discours de haine, la violence physique, autant de situations auxquelles les femmes font actuellement face, à l'instar de leurs prédécesseures pourchassées dans une Europe marquée par le flamboiement sinistre des bûchers à l'aube de la Renaissance. En effet, selon nombres de théoriciennes, écrivaines et historiennes, telles que Françoise d'Eaubonne ${ }^{4}$, Sylvia Federici $^{5}$, Barbara Ehrenreich ${ }^{6}$, la qualification de sorcière renvoyait généralement à « toute tête féminine qui dépassait ${ }^{7} \gg$ et, ainsi, dérangeait. La journaliste Mona Chollet, dans l'ouvrage Sorcières. La puissance invaincue des femmes, précise que « dans une logique familière aux femmes de toutes les époques, chaque comportement et son contraire [peut] se retourner contre vous ${ }^{8}{ }^{\prime}$. Du côté des artistes, Camille Ducellier écrit quand à elle en 2011 dans son introduction au Manifeste du féminisme divinatoire que «toute personne ayant l'intime conviction de s'écarter, d'une manière ou d'une autre, de la 'bonne femme contemporaine', descendante directe de la prénommée Ève, est invitée à se reconnaître disciple de Lilith ${ }^{9}$ ». C'est en substance ce que proclamaient cinquante ans plus tôt les membres de la W.I.T.C.H. (Women's International Terrorist Conspiracy from Hell) dans leur manifeste : "Inutile d'adhérer à WITCH. Si vous êtes une femme et que vous osez regarder à l'intérieur de vousmême, alors vous êtes une sorcière ${ }^{10}$.»

\footnotetext{
1. Ophélie Naessens : Université de Lorraine, Metz, France. ophelie.naessene@univ-lorraine.fr

2. Camille Ducellier, Sorcières, mes sœurs, https://www.camilleducellier.com/portfolio/sorcieres-mes-soeurs$\mathrm{dvd} /$.

3. Des images sont visibles ici : https://www.youtube.com/watch? $\mathrm{v}=4 \mathrm{aUd}-\mathrm{e}$ nozc

4. Françoise d'Eaubonne, Le sexocide des sorcières (Paris : L'Esprit frappeur, 1999).

5. Sylvia Federici, Caliban et la sorcière. Femmes, corps et accumulation primitive, trad. collectif Senonevero (Paris : Éditions Entremondes, [2004] 2017).

6. Barbara Ehrenreich and Deirdre English, Sorcières, sagesfemmes \& infirmières. Une histoirE des femmes soignantes, trad. L. Lame (Paris : Camourakis, [1973] 2014).

7. Mona Cholet, Sorcières. La puissance invaincue des femmes (Paris : Éditions La Découverte, 2018), 17.

8. Cholet, Sorcières. La puissance invaincue des femmes, 17.

9. Camille Ducellier, Le guide du féminisme divinatoire (Paris : Cambourakis, [2011] 2018), 22.

10. Manifeste de WITCH NY, 1968. "There is no joining W.I.T.C.H. .If you are a woman and dare to look within yourself, you are a witch." Margot Adler, Drawing Down the Moon: Witches, Druids, GoddessWorshipers and Other Pagans in America (London :Penguin [1979] 2006), 208.
} 


\section{Femmes, sorcières et politique}

Depuis la naissance des mouvements féministes aux États-Unis à la fin des années 1960, des femmes se sont manifestées comme productrices de rituels magiques. En 1968 à Halloween, le groupe W.I.T.C.H. fait sa première apparition dans les rues de Wall Street, maudissant avec force et fracas les rois de la finance new-yorkaise. Au-delà du nom choisi, ces femmes endossent les attributs vestimentaires et les accessoires culturellement associés aux sorcières : toutes de noir vêtues, encapées, défilant la tête coiffée de chapeaux, brandissant leurs balais. Davantage, les membres de la WITCH revendiquent l'usage de rituels à vocation politique. Plus précisément ici, celui-ci consistait en un sortilège à l'adresse du centre financier prenant la forme d'un chant sacré berbère. Robin Morgan, l'une des witches, raconte : "Les yeux fermés, la tête baissée, les femmes entonnèrent un chant berbère (sacré aux yeux des sorcières algériennes) et proclamèrent l'effondrement imminent de diverses actions. Quelques heures plus tard, le marché clôtura en baisse d'un point et demi, et le lendemain, il chuta de cinq points ${ }^{11}$. » Néanmoins, cette dernière n'hésite pas à avouer une méconnaissance de l'histoire des sorcières partagée avec ses acolytes : «À la Bourse, nous avons demandé une entrevue avec Satan, notre supérieur - un faux pas qui, avec le recul, me consterne : c'est l'Église catholique qui a inventé Satan et qui a ensuite accusé les sorcières d'être satanistes. Nous avons mordu à l'hameçon patriarcal sur ce sujet, et sur tant d'autres. Nous étions complètement stupides. Mais nous étions stupides avec du style ${ }^{12}$. " Par ailleurs, notons que si certaines des membres de WITCH ont cherché à étudier plus sérieusement la sorcellerie, pour la plupart d'entre elles, celle-ci ne relève pas d'une pratique avérée, mais bien d'un emprunt essentiellement iconographique. La forme de cette première apparition publique s'inspire explicitement du vocabulaire formel et gestuel de la manifestation politique : des femmes défilent dans la rue en procession, brandissant des slogans évocateurs. En manifestant dans le cœur financier des États-Unis, elles affirment un engagement anticapitaliste, associé à un engagement anti-patriarcal. En effet, associée aux revendications à destination des acteurs du marché boursier, les WITCH mènent une campagne anti-sexiste, visant, entre autres, les concours de beauté, le Playboy Club et l'institution du mariage. Les manifestantes cumulent ainsi une double revendication politique: anti-capitaliste et anti-patriarcale. Ce n'est d'ailleurs pas un hasard puisque certaines des membres étaient parallèlement reconnues comme activistes féministes, telle Heather Booth. Cette dernière était en effet engagée dans les mouvements des droits civiques (Civil Right Movements), le mouvement de libération des femmes à Chicago (Chicago Women's Liberation Union), et fut à l'origine de The Jane Collective, une organisation clandestine de conseil et d'accompagnement à l'avortement. WITCH propose ainsi de combiner l'iconographie de la sorcière avec les revendications radicales à l'époque - du mouvement de libération des femmes dans une visée à la fois divertissante, déroutante et politique.

Dans cette perspective anti-patriarcale et anti-capitaliste, comme, un peu plus tard, dans celle écoféministe menée par la figure incontournable de Starhawlk, des femmes engagées s'attachent à penser et mettre en œuvre des rituels susceptibles de produire une puissance collective en réaction à des situations qu'elles considèrent comme inacceptables (menace nucléaire, patriarcat, capitalisme, etc.). Dans l'introduction de l'ouvrage Reclaim. Recueil de textes éco-féministes, Émilie Hache explique l'ancrage et la fabrication de cette pensée mêlant enjeux féministes et écologiques au cœur des mobilisations : «- à travers la redécouverte de l'histoire de la destruction croisée, au cours de la modernité, des femmes et de la nature ; à travers la reconnaissance de points de passage entre la peur d'anéantissement devant

11. Robin Morgan, "WITCH hexes Wall Street", in Going Too Far. The Personnal Chronicle of a Feminist (New York: Random House/Vintage Paperbacks, 1977).

12. Robin Morgan, "Three articles on WITCH ", in Going Too Far. The Personnal Chronicle of a Feminist (New York: Random House/Vintage Paperbacks, 1977). 
l'utilisation du nucléaire et la peur quotidienne des femmes d'être insultées, agressées, violées; à travers encore la prise de conscience de l'importance de fabriquer de la confiance et de l'estime de soi pour espérer répondre à cette situation d'une manière sensible ${ }^{13}$ ». Les manifestations écoféministes recourant au vocabulaire iconographique sorcier sont aussi nombreuses, notamment dans le contexte des luttes anti-nucléaires des années 1980 aux USA, dont la plus connue est sans doute la Women Pentagon Action ${ }^{14}$. Cette dernière est considérée comme l'une des premières actions manifestant une tentative d'articuler féminisme, politique écologique et politique de paix. Cette initiative d'un groupe de militantes féministes également impliquées dans le mouvement anti-nucléaire découlait à la fois d'un événement particulier: une défaillance technique ayant entraîné la fonte d'une partie d'un réacteur nucléaire en Pennsylvanie, mais aussi, plus largement, de l'accélération de l'armement du pays et de la multiplication des catastrophes écologiques à travers le monde. Durant cette action, deux mille femmes convergent vers le Pentagone, déguisées et armées de marionnettes. La procession se déroulait selon plusieurs étapes: le deuil, la colère, l'empowerment et le défi ; les femmes avançant en tambourinant, chantant et tressant des « rubans de vie ». Elles se regroupaient finalement pour la lecture d'une déclaration commune dont voici un extrait :

« Nous nous réunissons au Pentagone, le 16 novembre parce que nous avons peur pour nos vies. Peur pour la vie de cette planète, notre Terre, et pour la vie de nos enfants qui sont le futur de notre humanité [...] Nous voulons mettre un terme à la course aux armements. Plus de bombes. Plus d'effarantes inventions de mort. [...] Nous savons qu'il existe une manière aimante, sensée et saine de vivre et nous avons l'intention de vivre de cette manière dans nos quartiers et dans nos exploitations agricoles de ces États-Unis, et parmi nos sœurs et frères de tous les pays du monde ${ }^{15} \gg$.

Dans sa préface à l'édition française de Dreaming the Dark de Starhawk, la philosophe belge Isabelle Stengers, à propos de la fête de Brigid organisée suite à l'élection de Reagan en 1981, parle du «premier rite collectif fabriqué délibérément pour faire converger politique et magie ${ }^{16}$ ». Parler de magie ici ne renvoie évidemment pas à l'idée de l'« intervention d'un pouvoir mystérieux et fascinant, surnaturel», mais «oser dire 'magie', c'est célébrer l'événement en tant que tel, c'est-à-dire le surgissement d'un possible, la sensation qu'a été défait quelque chose qui 'liait' la pensée et la vouait donc à l'impuissance. [...] Il n'y a aucune garantie ici [...], mais ce qu'on pourrait appeler une mise en indétermination, la création d'une inconnue qui fait bégayer les 'nous savons bien', qui ouvre les interstices par où se fait sentir la possibilité d'une autre histoire, même si cela reste une histoire improbable ${ }^{17} »$. Aussi, comme le soulignait Émilie Hache dans une intervention intitulée «Starhawk, le rituel et la politique » à l'occasion du cinquième Congrès Marx International à l'Université Paris-Sorbonne en 2007, associer l'action politique au rituel, considéré comme «un mouvement d'énergie organisé pour accomplir un but ${ }^{18}$ », permet d'envisager la politique comme une action susceptible de transformer les personnes qui y sont investies, et,

\footnotetext{
13. Émilie Hache, «Introduction. Reclaim Ecofeminism! », in Reclaim. Recueil de textes éco-féministes, ed. Émilie Hache (Paris : Cambourakis, 2016), 15-16.

14. Pour davantage d'informations sur cette action et son contexte d'émergence, le·a lecteur·trice peut se référer à l'article de Ynestra King «Si je ne peux pas danser, je ne veux pas prendre part à votre révolution », in Reclaim. Recueil de textes éco-féministes, ed. Émilie Hache (Paris : Cambourakis, 2016), 105-126.

15. Ynestra King « Si je ne peux pas danser, je ne veux pas prendre part à votre révolution », in Reclaim. Recueil de textes éco-féministes, ed. Émilie Hache (Paris : Cambourakis, 2016), 115.

16. Isabelle Stengers « Postface. Un autre visage de l'Amérique? », in Starhawk Rêver l'obscur. Femmes, magie et politique (Paris : Cambourakis, 2015), 362.

17. Stengers « Postface. Un autre visage de l’Amérique ? «, 362-363.

18. Starhawk Rêver l'obscur. Femmes, magie et politique, 234.
} 
davantage, le monde qui les entoure, une action politique qui « nous [change] profondément $[\ldots]$ parce que notre transformation est intégrée à la transformation de la réalité ${ }^{19}{ }$.

\section{"Witch blocks " aujourd'hui}

Dans les sociétés contemporaines, des activistes - associé·e·s ou non à des artistes participent à la résurgence de la perspective militante du rituel. Aux États-Unis par exemple, des sorcières participent au mouvement Black Lives Matter, protestant contre l'épidémie de meurtres racistes perpétrés par la police dans nombres d'états. Aussi, depuis 2017, des sorcières et sorciers du monde entier jettent un sortilège destiné à envoûter le président Donald Trump, répétant le sort collectif à chaque lune descendante. En France également, sorcières et sorciers viennent grossir les rangs des manifestations (le Labofii - The Laboratory of Insurrectionary Imagination, le Stras - Syndicat du Travail Sexuel, les Witch Blocs, la Cellule d'Action Rituelle à la Z.A.D. de Notre Dame des Landes, etc.). À travers cette résurgence, l'imaginaire de la sorcière est plus que jamais ravivé, et nous assistons aujourd'hui à ce que certaines considèrent comme une deuxième vague $\mathrm{WITCH}^{20}$. En septembre 2017 en France, pendant le large mouvement de contestation des nouvelles réformes du code du travail ${ }^{21}$ ), le Witch Bloc Paname ${ }^{22}$ fait son entrée en scène, au rythme des «Macron au chaudron! ", «Macron au bûcher!", «Conservatisme, du balai ! " ${ }^{23}$. À nouveau, des manifestantes grimées envahissent les rues. Outre le fait que la figure de la sorcière soit devenue une emblème féministe depuis la fin des années 1960, les membres du Witch Bloc Paname expliquent leur choix de cette réappropriation : «En tant qu'archétype, elle symbolise des siècles d'histoire patriarcale, puisqu'à travers les âges, les femmes qui étaient savantes, notamment en médecine naturelle, ou simplement indépendantes des hommes étaient qualifiées de 'sorcières' et chassées, torturées, tuées. Nous réclamons donc ce titre pour montrer l'existence de nos luttes et notre détermination à les mener ${ }^{24} . »$ Le collectif se revendique comme groupe anonyme, féministe, radical, antiraciste et non-mixte. À l'instar de leurs précurseuses, nous retrouvons chez les parisiennes la reconquête des codes de l'oppression ainsi qu'un désir de concevoir la lutte contre toutes les formes de domination par le biais de l'action collective. À ces principes, elles ajoutent celui de l'intersectionnalité, associée à une réaction contre " les pratiques virilistes présentes dans les cortèges du black bloc » se traduisant par la création d'un cortège non-mixte, "un espace pour militer collectivement entre féministes radicales, et ce sans présence d'hommes cisgenres, nonconcernés par les oppressions du patriarcat ${ }^{25} \gg$. Comme les membres de la WITCH des années 1960, si de véritables praticiennes de sciences occultes se comptent dans les franges de ce «bloc ${ }^{26}$, toutes ne se retrouvent pas dans l'exercice magique. Ces nouveaux witch blocs

19. Starhawk Rêver l'obscur. Femmes, magie et politique, 244.

20. Heather Booth, "Wicked W.I.T.C.H.:The 60s Feminist Protestors Who Hexed Patriarchy", Vice, 2016, consulté le 23 novembre 2019, https://www.vice.com/en_us/article/43gd8p/wicked-witch-60s-feministprotestors-hexed-patriarchy.

21. Cette réforme consistait entre autres à un affaiblissement du pouvoir des salariés, à une facilitation des conditions de licenciement, une augmentation des contributions sociales ainsi qu'une baisse de l'aide au logement.

22. Expression argotique pour désigner Paris.

23. Des images visibles ici : https://manifesto-21.com/feminisme-sorcellerie-luttes-rencontre-witch-bloc-deparis/

24. Witch Bloc Paname, «Féminisme, sorcellerie et luttes. Rencontre avec le witch bloc de Paris » interview par Laure Boussat, Manifesto XXI, février 2018, https://manifesto-21.com/feminisme-sorcellerie-luttes-rencontrewitch-bloc-de-paris.

25. Witch Bloc Paname, «Féminisme, sorcellerie et luttes. Rencontre avec le witch bloc de Paris ».

26. Dans une interview avec Céline du Chéné, la représentante du Witch Bloc Marseille déclare que, selon elle « être sorcière signifie pratiquer la magie », et revendique ainsi « un affranchissement à l'égard de la société, des 
essaiment aujourd'hui dans toute la France, et leurs revendications politiques épousent les thèmes d'actualité : droit à l'avortement, Procréation Médicalement Assistée, violences faites au femmes, urgence climatique, entremêlant revendications féministes et écologistes. La recrudescence actuelle d'une lutte active contre les violences faites aux femmes s'est logiquement pris pour étendard la sorcière, laquelle est devenue depuis les relectures féministes de l'histoire médiévale ${ }^{27}$, une victime emblématique des violences faites aux femmes - violence physique, psychologique, sociale. Outre les traditionnels dress code noir et chapeaux pointus, ces manifestations publiques reprennent également des formes et des images issues des savoirs et pratiques sorciers. La principale d'entre elles repose sur la constitution d'un cercle magique, «apparai[ssant] comme un moyen intense de lutter contre une douleur, une peur, une injustice, un fantôme ${ }^{28}$ ». Déjà en 1982 dans Dreaming The Dark, Starhawk explicitait le rôle et l'intérêt du cercle dans les rituels :

«Les structures de l'immanence sont circulaires : clans, tribus, convents, collectifs, groupes de soutien, groupes d'affinités, groupes de prise de conscience sont des cercles. Dans un cercle, le visage de chaque personne peut être vu, la voix de chaque personne peut être entendue et appréciée. Tous les points du cercle sont équidistants de son centre; c'est sa définition et sa fonction : distribuer l'énergie de manière égale ${ }^{29}$. »

Ces femmes militantes conçoivent à destination de l'espace public des exemples de résistance créative inspirés de pratiques rituelles plus anciennes et de l'iconographie sorcière, et ce dans différentes perspectives de combat (féministe, écologiste, altermondialiste, etc.). En cela, ces initiatives rejoignent en partie les ambitions des partisans de l'art-activisme pour lesquels les actions menées doivent associer caractéristiques esthétiques et politiques. Esthétique, puisque celles-ci sont pensées dans une attention à la forme de la résistance, et politique, puisqu'il s'agit bien d'agir sur une réalité identifiée comme problématique. À travers l'action esthétique, il s'agit pour les artactivistes de donner envie d'agir, de rendre la lutte désirable, parce que plus libre et créative. En cela, les membres des witch blocs participent à transformer la politique en matière artistique, au sens où l'entend Isabelle Frémeaux, cofondatrice du Laboratoire d'Imagination Insurrectionnelle ; « les actions politiques deviennent des actions poétiques, esthétiques, qui touchent le sensible, des questions de beauté, les mouvements sociaux en sont les matériaux ${ }^{30} \gg$.

\section{Artistes et sorcières : de la possibilité de nouveaux récits}

Dans le champ des arts visuels, les années 1970 sont également marquées par un (ré-) investissement et un renouvellement des pratiques rituelles accompagnés d'une réappropriation de l'iconographie des sorcières. En 1977, tandis qu'est découvert un nouveau corps de victime des "Hillside Stranglers », un duo de serial killers ayant violé et tué dix femmes entre 1977 et 1979, les artistes Suzanne Lacy et Leslie Labowitz décident d'exprimer leur deuil, leur peur et leur colère en formulant à l'occasion d'une performance publique une critique féministe du traitement spectaculaire de ces meurtres. En effet, à chaque nouvelle victime découverte, l'intérêt des médias s'accroissait, et la couverture des faits n'échappait ni au sensationnalisme ni à l'objectivisation des corps féminins.

sciences et de la religion judéo-chrétienne ». Céline du Chéné, Les sorcières. Une histoire de femmes, (Paris: France Culture, 2019), 161.

27. Barbara Ehrenreich and Deirdre English, Sorcières, sagesfemmes \& infirmières. Une histoirE des femmes soignantes, trad. L. Lame (Paris : Camourakis, [1973] 2014).

28. Julie Crenn, «White Blood, Blue Night : Les sorcières s’invitent au CAC La Traverse », Friction magazine, consulté le 23 novembre 2019, https://friction-magazine.fr/white-blood-blue-night-sorcieres-sinvitent-cactraverse.

29. Starhawk, Rêver l'obscur. Femmes, magie et politique, 182.

30 . Isabelle Frémeaux, «Art-activisme», France Culture, accessed November 17, 2019,https://www.franceculture.fr/emissions/terre-terre/l-art-activisme. 


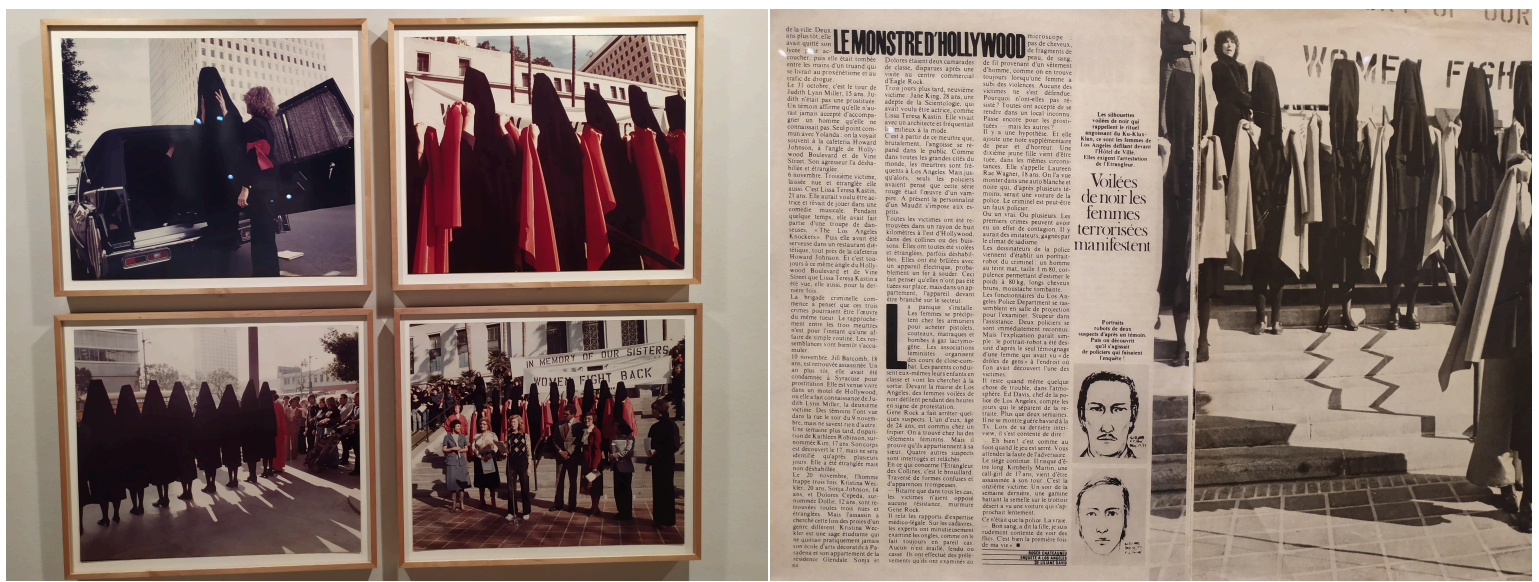

Figures 1 et 2. Suzanne Lacy et Leslie Labowitz, In Mourning and in Rage, 1977.

Pour la performance In Mourning and In Rage, dix femmes se sont réunies sur le parvis de la mairie de Los Angeles, revêtues de toges dissimulant leur visage. Une par une, chaque femme déclarant «Je suis ici pour les dix femmes qui ont été violées et étranglées entre le 18 octobre et le 19 novembre ». In Mourning and In Rage visait en outre à relier le cas singulier de ces féminicides californiens à une image plus large de la violence à l'égard des femmes à l'échelle nationale, tout en invitant celles-ci à une action collective transformatrice «IN MEMORY OF our sisters, we fight back ! ${ }^{31} »$.

L'intérêt pour l'imaginaire de la sorcellerie n'a pas disparu chez les artistes contemporaines, et revêt des formes diverses allant de la revendication spectaculaire à la convocation de dispositifs et d'images. Gangs of Witches, par exemple, se présente comme un collectif d'artistes (art gang) "féministe, intersectionnel, écologiste», réunissant arts plastiques, musique et arts vivants, et dont le fait d'armes le plus représentatif est le festival « Patriarchy is burning » qui s'est tenu en juin 2019 dans le musée d'art contemporain du Palais de Tokyo à Paris. Accompagné d'un livre et d'un album éponymes, le festival se présentait comme tel : " Gang Of Witches réunit une quinzaine d'artistes, peintres, sculpteur.rice.s, écrivain.e.s, photographes, vidéastes, musicien.ne.s, danseur.se.s et performeur.se.s, et s'apprête à envoyer le patriarcat au bûcher ${ }^{32}$.» Les membres du Gang expliquent leur choix de la figure de la sorcière, renvoyant explicitement à la WITCH des années 1960 : "L'image de la sorcière, savante, indépendante et puissante, souvent crainte, parfois moquée, toujours auréolée de mystère et maîtresse de son identité, est un marqueur de la place des femmes dans la société et des enjeux de chaque époque. Elle est, depuis les années 1960, une icône féministe, écologiste, anticapitaliste, et le symbole idéal pour le gang $^{33}$. » $\mathrm{Au}$ premier regard, la convocation de la sorcière semble ici gouverner des choix stylistiques, tant en matière de communication visuelle que d'apparence vestimentaire ${ }^{34}$. Néanmoins, au-delà de la simple apparence des performeur·e's et autres artistes présents, la sémiotique de l'événement fait aussi la part belle à son imaginaire, les thématiques des œuvres abordent par exemple l'entrée du monde dans un nouvel âge sombre, lequel renverrait à celui du sexocide des sorcières

31. Malheureusement, l'action des artistes n'a pas échappé à une interprétation médiatique erronée, des journalistes les qualifiant de «femmes terrorisées », dont « les silhouettes voilées de noir rappellent le rituel angoissant du Ku-Klux-Klan ». Cf. Figures 1 et 2. Suzanne Lacy et Leslie Labowitz, In Mourning and in Rage, 1977. Photographies prises par l'auteure à l'occasion de l'exposition Feminisms !, Centre de Cultura Contemporania de Barcelona, juillet 2019.

32. Gang of witches, « Patriarchy is burning », consulté le 13 octobre

2019,https://www.gangofwitches.com/what-we-do/patriarchy-is-burning/

33. Gang of witches, « Who we are », consulté le 13 octobre 2019, https://www.gangofwitches.com/who-we-are/

34. Gang of witches, « Patriarchy is burning ». 
(Mina Mond, Les nouveaux Cavaliers de l'Apocalypse), le bûcher (Aron Demetz Burning, Burning Man), le rapport à la terre mère, convoquant ainsi les perspective éco-féministes

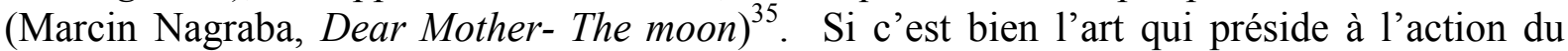
gang, ses membres revendiquent aussi un engagement, " au service d'une légitime soif de justice », et précisent que les « œuvres font écho aux foyers de résistance qui s'organisent contre la fascisation du monde ${ }^{36} \|$, invitant ainsi à la révolte. Aussi, le gang revendique une approche intersectionnelle de la lutte, en abordant, je cite « les thèmes des violences faites aux femmes, de la masculinité libérée des injonctions patriarcales, et interroge les codes du genre pour une prise de conscience globale vers plus d'égalité et de diversité ${ }^{37}$ ».

Si la démonstration d'une affiliation à la figure tutélaire de la sorcière confine parfois à un aspect communicationnel spectaculaire, voire, à un effet de mode, pour d'autres artistes, l'iconographie de la sorcellerie se manifeste davantage sous forme d'images mentales, d'une poétique de l'incantation. L'artiste pluridisciplinaire Myriam Minhidou présente régulièrement des performances, souvent troublantes pour leur public, qu'elle nomme «transperformances », n'hésitant pas à qualifier son travail d'artiste de chamanique. Ses pièces sont conçues comme des rituels à visée cathartique, des «moments de mise à l'épreuve de son corps pour transcender un trauma, une violence, une blessure ${ }^{38} \gg$.

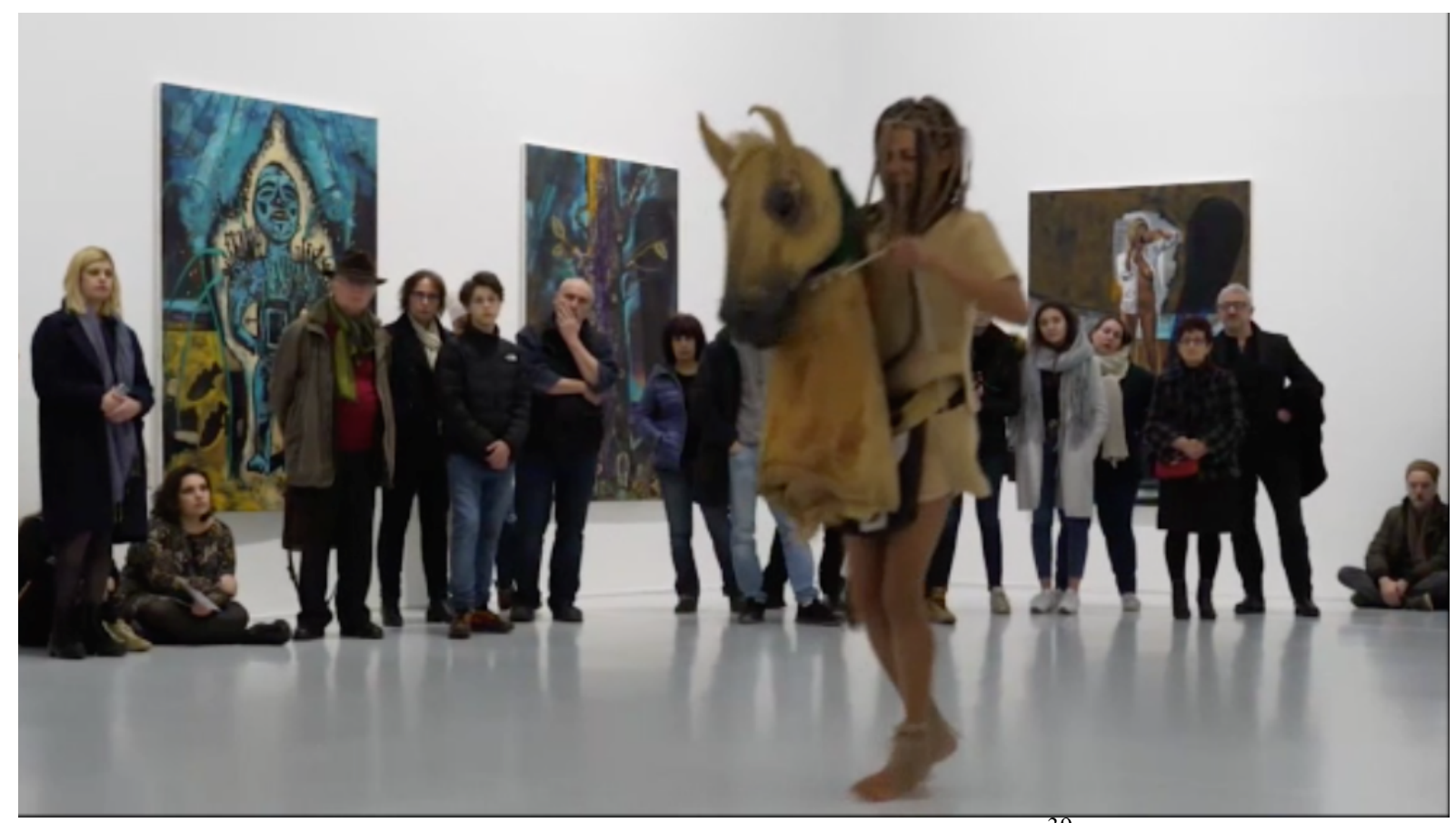

Figure 3. Myriam Minhidou, Galop, $2019^{39}$.

En février 2019, dans le contexte d'une exposition consacrée à Damien Deroubaix, l'artiste présente au musée d'art moderne et contemporain de Saint Étienne la performance Galop, accompagnée à la voix par Mariette Auvray. Dans cette pièce qui, selon son auteure, parle

35. Gang of witches, « Communiqué de presse », consulté le 13 octobre 2019, https://www.gangofwitches.com/wpcontent/uploads/2019/05/05_19_communique_de_presse_GOW_PIB_HD_FR.pdf

36. Gang of witches, "Communiqué de presse ».

37. Gang of witches, « Communiqué de presse ».

38 . Julie Crenn, « Myriam Minhidou », Aware, consulté le 20 novembre 2019, https://awarewomenartists.com/artiste/myriam-mihindou/

39. Capture d'écran, reportage en ligne : https://www.arte.tv/fr/videos/085905-006-A/myriam-mihindou/ 
d' « énergies, d'indocilité, d'oracle, de mythe ${ }^{40} »$, et de réparation d'un traumatisme féminin, le travail de la performeuse consiste ici à incorporer le chant pour animer son corps de femme, lequel devient progressivement celui d'un équidé, ainsi qu'à «puiser une énergie mystique mais profonde de manière à ce que l'oracle puisse s'annoncer ${ }^{41} \gg$. Dans une interview accordée à la revue Mouvements, Myriam Minhidou explique qu' " [elle] tien[t] à produire des œuvres qui mène à des perspectives intérieures pour entrer en connexion avec les

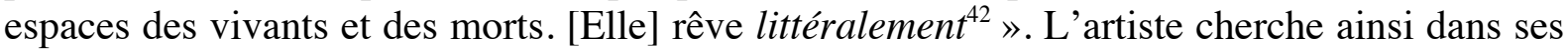
performances à produire des œuvres méditatives, des transes susceptibles d'ouvrir à l'écoute de soi-même et du monde qui nous entoure. Néanmoins, ces sorties corporelles ne relèvent pas ici d'une finalité, mais, davantage, elles visent à permettre l'incarnation des maux et des blessures, tels que l'oppression, la violence, les luttes de pouvoir, que l'artiste se donne pour ambition d'apaiser. En se plaçant ainsi en posture de celle qui est susceptible de réparer les mauvais sorts, nous suggérons que la pratique de Myriam Minhidou pourrait se penser à travers le prisme d'une pensée du care. Dans son ouvrage Moral Boundaries : A Political Argument for an Ethic of Care, la philosophe canadienne Joan Tronto définit ainsi le care :

" En règle générale, on peut dire que le care est une activité propre à notre espèce qui regroupe toutes les actions destinées à entretenir, réparer et faire perdurer notre "monde" afin d'y vivre dans les meilleures conditions possibles. Ce monde comprend notre corps, notre soi et notre environnement, trois éléments que nous tentons d'entremêler en une toile complexe et vitale $^{43}$.»

Nous suggérons par conséquent ici que certains rituels sorciers s'apparentent à des rituels de « réparation », et que les pratiques magiques articulent toujours corps physique, soi et Nature. Aussi, contrairement à la perspective prônée par la sorcellerie néopaïenne, le terme de sorcellerie employé par les féministes renvoie à une identification aux femmes persécutées, et est ainsi associé à un processus de guérison.

À l'occasion de White Blood, Blue Night (CAC La Traverse, 2017), Myriam Minhidou rejoint le groupe de «sorcières » convoquées par Julie Crenn, commissaire de l'exposition. Si les sorcières furent et sont toujours exclues de la société, en marge, c'est avant tout à travers leur potentiel à remettre en cause l'ordre établi. Selon Julie Crenn, «Depuis l'Antiquité, les sorcier'e·s sont littéralement des 'diseur'se's de sorts'. Leur existence et leur représentation cristallisent une peur collective intrinsèquement liée à celle d'une perte de pouvoir, à la possibilité d'une déstabilisation du groupe dominant ${ }^{44}$. " Chez Myriam Minhidou, le «souci des autres " est intrinsèquement lié à une dimension politique dans la mesure où l'artiste construit des espace de résilience qui sont tout autant des espaces de résistance, vis-à-vis des formes d'oppression et des violences subies par les femmes et les personnes racisées.

Nous poursuivons ici par un second exemple de pratique artistique contemporaine que nous avons eu l'occasion d'accueillir à la Galerie 0.15//Essais Dynamiques - Espace d'art et de création de l'Université de Loraine, sur le site de l'île du Saulcy à Metz. En novembre 2018, nous y présentions le travail de Tatiana Karl Pez, artiste protéiforme - performeuse, poétesse, sculptrice, chanteuse, vidéaste, etc. Dans le cadre du programme curatorial R.A.A.C. (Retour de l'Affect dans l'Art Contemporain), elle proposait une exposition nommée Tu brûles. Paradigma $>$ Pleistocen $>$ Paradismissed, inaugurée par la performance Danse de la main

40. Myriam Minhidou, L'Atelier A, Arte TV, consulté le 20 novembre 2019, https://www.arte.tv/fr/videos/085905-006-A/myriam-mihindou/

41. Myriam Minhidou, L'Atelier A, Arte TV.

42. Myriam Minhidou, interview par Sylvie Arnaud, revue Mouvements, 3 avril 2018, en ligne: http://www.mouvement.net/teteatete/entretiens/myriam-mihindou.

43. Joan Tronto, Moral Boundaries : A Political Argument for an Ethic of Care (New York : Routledge, 1993), 103

44. Julie Crenn «White Blood, Blue Night», Friction Magazine, consulté le 23 novembre, 2019, en ligne : https://friction-magazine.fr/white-blood-blue-night-sorcieres-sinvitent-cac-traverse/ 
négative. La performance se déroulait dans un espace plongé dans la pénombre. Pour nous spectateurs, il semblait que nous y pénétrions après-coup, plausiblement à la suite d'une catastrophe naturelle. Incendie ? Sécheresse ? Passage d'un être humain? Non-humain ? De ce temps pré-apocalyptique, un «alter » nous adressait un message, constitué de sons, de gestes, d'images et d'émotions dont l'artiste se faisait la médiatrice. Cette singulière correspondance nous était transmise au prisme d'un habile et poétique jeu de réflexion de lumières naturelles et artificielles, lequel esquissait les contours d'une scène imaginaire, telle le souvenir d'un rituel immémorial, d'une nuit lointaine.

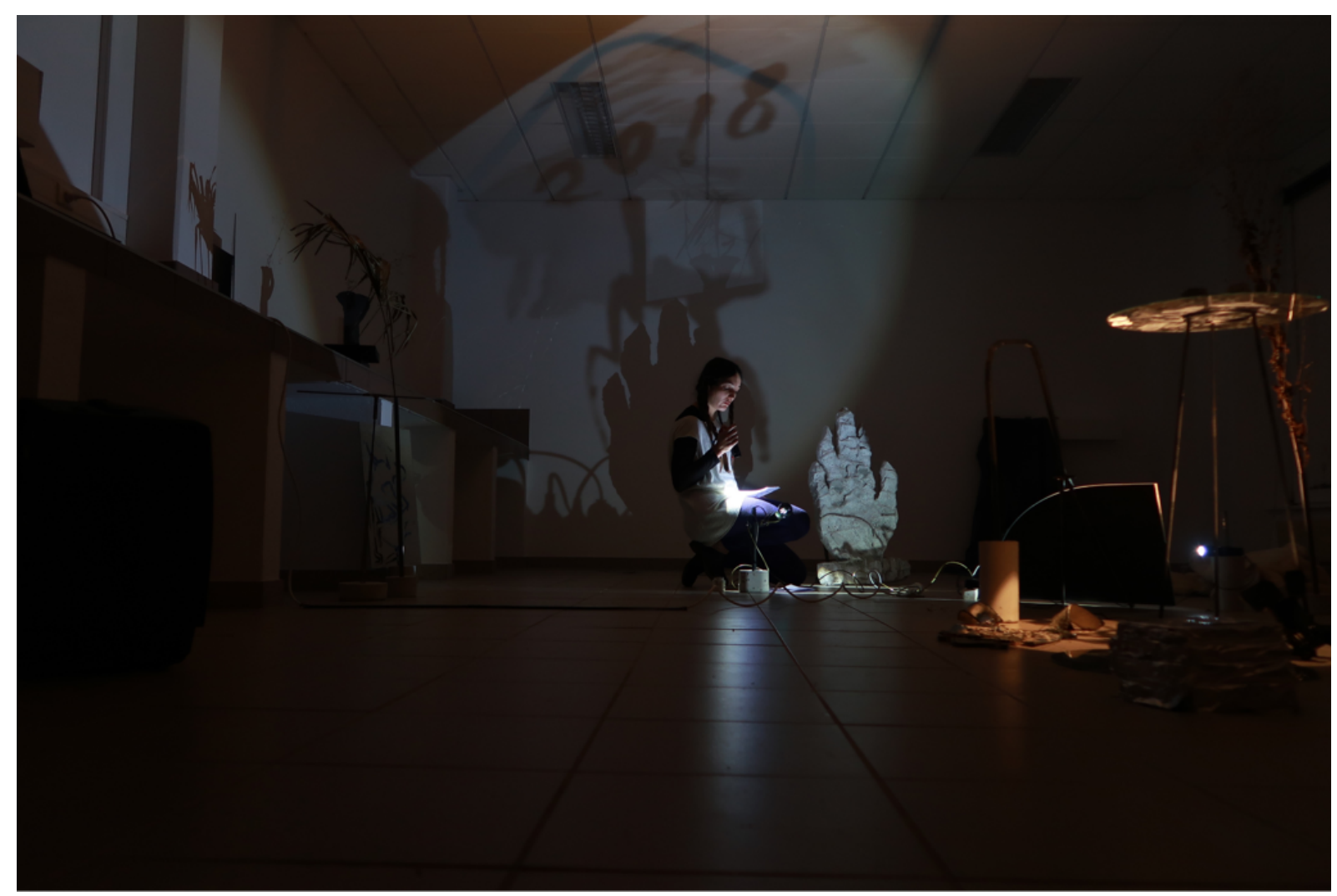

Figure 4. Tatiana Karl Pez, Danse de la main négative, 2019. (Performance)

Le titre de l'exposition référait explicitement au Pleistocène, première époque géologique du Quaternaire marquée par d'intenses périodes de glaciation de la Terre. Actuellement, le permafrost ne cesse de se réduire, dévoilant minéraux, fossiles, plantes et corps autrefois enfouis. À l'image de la situation géologique contemporaine, dans l'installation, avec le dégel, les fleurs (re-)surgissaient ${ }^{45}$. Le travail de Tatiana Karl Pez relève d'une attention précise et incarnée à la nature, nourrie par une connaissance des plantes et de la terre, laquelle participe à une remise en cause des différentes formes d'exploitation du vivant ainsi que des ressources naturelles. Au cours de la performance, l'artiste devenait maîtresse de cérémonie, ses sabots en bois claquaient sur le sol et son chant s'élevait dans le crépuscule ; un chant faisant écho tant aux ritournelles médiévales qu'aux mélodies mystérieuses fredonnées par nos grand-mères. Ici, le rituel tribal célébrait le surgissement d'un possible. L'installation de Tatiana Karl Pez, sans éléments factuels pour concevoir notre «Paradis perdu» (Paradismissed), nous invitait à ressentir la possibilité d'une autre histoire pour notre planète, à nous souvenir d'un temps oublié, ou, à défaut, à l'imaginer. Dans un geste collectif de résistance, Tatiana Karl Pez nous enjoignait à venir fleurir les terres brûlées. 
Si les savoirs et les pratiques sorciers induisent des pouvoirs tels que celui de guérir (Myriam Minhidou) ou de deviner (Tatiana Karl Pez), les gestes et rituels mis en place par ces deux artistes, à travers une multiplicité d'énergies que leurs performances propagent, dessinent des postures de résistance traversant tant les perspectives féministes, écologiques que curatives. En racontant de nouvelles histoires, et en libérant les imaginaires, les artistes convoquées ouvrent des possibilités de déstabilisation des groupes et des modèles discursifs dominants, en proposant des récits potentiels pensés comme outils d'émancipation. En cela, elles se révèlent " diseuses de sorts », participant à l'élaboration d'un imaginaire qui se dresse contre les diverses formes de domination ou de violence, la magie devenant alors un instrument créatif de lutte. Pour conclure, nous retiendrons ici les mots écrits en 1982 par Starhawk :

«La magie a souvent été pensée comme l'art de faire devenir vrais les rêves : l'art de réaliser des visions. Mais avant de rendre réelle une vision, nous devons la voir. Nous devons donc avoir de nouvelles images à l'esprit, nous aventurer dans un paysage transformé, raconter de nouvelles histoires ${ }^{46}$. $»$

\section{Bibliographie}

Adler, Margot. Drawing Down the Moon:Witches, Druids, Goddess-Worshipers and Other Pagans in America. London :Penguin, [1979] 2006.

Cholet, Mona. Sorcières. La puissance invaincue des femmes. Paris : Éditions La Découverte, 2018.

Colin, Anne (dir.). Sorcières, pourchassées, assumées, puissantes, queer. Montreuil : édition B42/Maison populaire, 2012.

Crenn, Julie. «White Blood, Blue Night: Les sorcières s'invitent au CAC La Traverse ». Friction magazine, consulté le 23 novembre 2019. https://friction-magazine.fr/white-bloodblue-night-sorcieres-sinvitent-cac-traverse.

D’Eaubonne, Françoise, Le sexocide des sorcières. Paris : L'Esprit frappeur, 1999.

Ducellier, Camille. Le guide du féminisme divinatoire. Paris : Éditions Cambourakis, [2011], 2018.

Du Chéné, Céline. Sorcières. Une histoire de femmes. Paris : France Culture, 2019.

Ehrenreich, Barbara and English, Deirdre. Sorcières, sagesfemmes \& infirmières. Une histoirE des femmes soignantes. Traduit par L. Lame. Paris : Camourakis, [1973] 2014.

Federici, Sylvia. Caliban et la sorcière. Femmes, corps et accumulation primitive. Traduit par le collectif Senonevero. Paris : Éditions Entremondes, [2004] 2017.

Fremeaux, Isabelle. «Art-activisme », France Culture, consulté le 17 novembre 2019, https://www.franceculture.fr/emissions/terre-terre/l-art-activisme.

Hache, Émilie. « Starhawk, le rituel et la politique. Faire de la politique autrement, refaire de la Politique ", 2007, consulté le 23 novembre 2019, http://ecole-lacanienne.net/wpcontent/uploads/2016/04/Hache-Starhawk-le-rituel-et-la-politique.pdf

Hache, Émilie. Reclaim. Recueil de textes éco-féministes. Paris : Cambourakis, 2016.

46. Starhawk, Rêver l'obscur, 129. 
Morgan, Robin. Going Too Far. The Personnal Chronicle of a Feminist. New York : Random House/Vintage Paperbacks, 1977.

Starhawk. Rêver l'obscur. Femmes, magie et politique. Paris : Cambourakis, [1982] 2015.

Vice. "Wicked W.I.T.C.H.:The 60s Feminist Protestors Who Hexed Patriarchy". Dernière modification le 28 octobre, 2016. https://www.vice.com/en_us/article/43gd8p/wicked-witch60s-feminist-protestors-hexed-patriarchy.

Witch Bloc Paname. «Féminisme, sorcellerie et luttes. Rencontre avec le witch bloc de Paris ». Interview par Laure Boussat. Manifesto XXI, février 2018. https://manifesto21.com/feminisme-sorcellerie-luttes-rencontre-witch-bloc-de-paris. 